\title{
Research Paper \\ Capabilities and Requirements of the Elderly Remote Health Monitoring
}

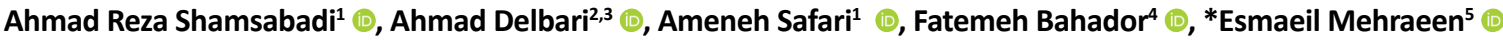

1. Department of Health Information Technology, Esfarayen Faculty of Medical Sciences, Esfarayen, Iran.

2. Iranian Research Center on Aging, University of Social Welfare and Rehabilitation Sciences, Tehran, Iran.

3. Division of Clinical Geriatrics, Department of Neurobiology, Care Sciences, and Society, Karolinska Institutet, Stockholm, Sweden.

4. Department of Health Information Technology, School of Ferdows Paramedical, Birjand University of Medical Science, Birjand, Iran.

5. Department of Health Information Technology, Khalkhal University of Medical Sciences, Khalkhal, Iran.

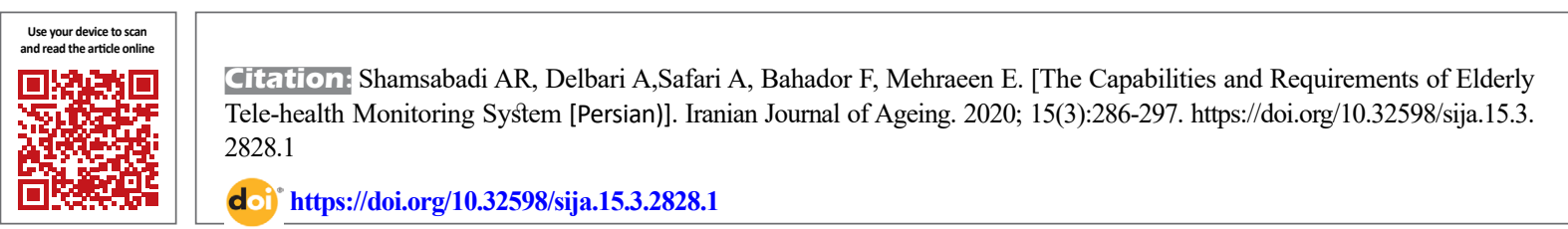

\section{(i) (8)}

Received: 03 nov 2019

Accepted: 17 may 2020

Available Online: 01 Oct 2020

Key words:

System, Remote

health monitoring,

Home care, Elderly,

Validation

\section{ABSTRACT}

Objectives Telehealth management systems for elderly people should have the features, capabilities, and infrastructure that meet home care management's diverse needs. Since there is no valid and reliable questionnaire to determine these requirements, this study's primary purpose was to determine a telehealth monitoring system's requirements for the elderly and present it as a valid and reliable questionnaire.

Methods \& Materials This study was descriptive-analytical and performed in 2019. The items for the Questionnaire were obtained from a systematic review study. To review, the selected keywords such as elderly, home care, telecare, telehealth, and information technology from 2013 to October 2019 were searched on selected databases.

Results Based on the study's findings, the parameters, and requirements of a telehealth monitoring system for the elderly were identified in 97 items. Furthermore, the first 31 items of the questionnaire were removed due to low validity and reliability. In the next phase, the final questionnaire with 66 items, content validity ratio of $99 \%$, Cronbach's alpha coefficient (0.9) and desirable correlation coefficient $(r=0.85$, $\mathrm{P}<0.05$ ) were designed.

Conclusion In this study, the requirements of a telehealth monitoring system for the elderly were determined, and a valid and reliable questionnaire was developed using these requirements. The questionnaire was designed in five parts: applications, functional requirements, non-functional requirements, tools, and communication infrastructure. Health care system designers may use the study results to determine the applications and requirements of elderly health monitoring systems.

\section{Extended Abstract}

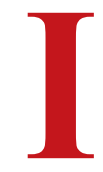

\section{Introduction}

$\mathrm{n}$ recent years, with the increase in the elderly population worldwide [1-4], human societies face an increasing prevalence of fragility in the elderly [5]. This fragility leads to other consequences such as dependence, chronic dis-

eases, reduced quality of life, long-term hospitalization, and increased care costs for the elderly [6-11]. Today, to manage and reduce fragility in these people, a new technology called elderly remote health monitoring is used, increasing significantly to help the elderly [12]. This technology's main aim is to reduce fragility and the length of hospital stay, and the cost of treatment for the elderly $[13,14]$. Given the importance of determining the requirements of remote care systems, the present

\section{* Corresponding Author:}

Esmaeil Mehraeen, PhD.

Address: Department of Health Information Technology, Khalkhal School of Medical Sciences, Khalkhal, Iran.

Tel: +98 (45) 32426801

E-mail: es.mehraeen@gmail.com
} 
study was conducted to determine the capabilities and needs of elderly $\mathrm{c}$ health monitoring.

\section{Methods \& Materials}

This study was a descriptive-analytical that was conducted in 2019 in two main stages. In the first stage, a review study was conducted to identify the capabilities and requirements of elderly remote health monitoring. At this stage, the keywords aging, home care, remote monitoring, remote health, and information technology were searched in the following scientific databases and the period 2013 to October 2019: PubMed, Scopus, Web of Science, Google Scholar.

The information items resulting from the comprehensive search of scientific databases were designed in a questionnaire. The questionnaire consisted of five parts and 97 questions reviewed and approved by a geriatrician and two health information management specialists. In the second stage, the validity and reliability of the designed questionnaire were examined. At this stage, the questionnaire was completed by ten specialists in geriatrics and health information management, and the following formula was used to evaluate its validity (Formula 1):

1.

$$
C V R=\frac{n e-n / 2}{n / 2}
$$

In this regard, "ne" is the number of specialists who have answered the "necessary" option, and $n$ is the total number of specialists who have completed the questionnaire. In this study, the internal consistency method (Cronbach's alpha) was used to assess items' reliability. The retest test method (Spearman-Brown coefficient) was used to evaluate the reliability of the correlation between two components of a scale with a time and space interval.

\section{Results}

Based on the Results of the first phase of the study, 1738 articles were retrieved from searches in databases to identify the capabilities and requirements of elderly remote health monitoring. After deleting duplicate articles $(\mathrm{n}=468)$, articles that were not in English $(n=380)$, and also articles irrelevant to the purpose of the research $(n=858)$, finally, 32 articles were selected. The capabilities and requirements of elderly remote health monitoring were identified in five parts and 97 items by reviewing related articles. The identified capabilities and requirements were: system applications $(n=18)$, functional requirements $(\mathrm{n}=19)$, non-functional require- ments $(n=27)$, types of tools $(n=18)$, and communication infrastructure $(n=15)$.

The content validity calculation Results in the first step showed that out of 97 items of the questionnaire completed by 5 experts, 51 items the content validity ratio values were lower than $99 \%$. In the second step of calculating, content validity was recalculated by referring to the relevant experts, providing information, discussing the items, and getting their final opinions. Finally, out of 97 identified items, 69 items with a content validity ratio of over $99 \%$ were approved as capabilities and requirements of the elderly remote health monitoring. As a result, the approved capabilities and requirements were: system applications $(n=12)$, functional requirements $(n=15)$, nonfunctional requirements $(n=14)$, types of tools $(n=13)$, and communication infrastructure. $(\mathrm{n}=15)$ (Table 1$)$.

Cronbach's alpha coefficient was used to evaluate the questionnaire's internal reliability, calculated to be 90 among 15 geriatricians and 15 health information management specialists. Also, to assess the questionnaire's external reliability, the retest test method was used in which 10 experts completed the questionnaire in two stages with an interval of one week, and the correlation coefficient was 0.85 .

\section{Conclusion}

In this study, the remote monitoring system's capabilities and requirements for the health of the elderly in five parts of system applications, functional needs, nonfunctional requirements, tools, and communication infrastructure were identified and validated. The specified requirements are in line with the culture and technology, and communication infrastructure in Iran. Therefore, the use of these capabilities and requirements by remote health system designers will better understand the needs of vulnerable elderly in various dimensions. However, further research is needed to identify the identity and clinical information requirements of the elderly remote health monitoring. One of the limitations of this study is the low sample size to determine validity and reliability. It is suggested that in future studies, the validity and reliability of the questionnaire in a larger sample size be investigated using factor analysis. 
Table 1. Approved capabilities and requirements for the elderly remote health monitoring

\begin{tabular}{|c|c|c|c|c|c|}
\hline No. & System Applications & Functional Requirements & $\begin{array}{c}\text { Non-functional Require- } \\
\text { ments }\end{array}$ & Types of Tools & $\begin{array}{l}\text { Communication } \\
\text { Infrastructure }\end{array}$ \\
\hline 1 & Blood pressure monitoring & Ability to provide training & $\begin{array}{l}\text { Having a central database of } \\
\text { information }\end{array}$ & Clinical sensors & Internet \\
\hline 2 & Heart rate monitoring & $\begin{array}{l}\text { Recognize the urgency of the } \\
\text { elderly condition }\end{array}$ & User friendly & Camera & WiFi and WiMax \\
\hline 3 & Saturated oxygen level & Provide reminders & Based on the web & Tablet & SMS \\
\hline 4 & Weight monitoring & Ability to provide alerts & Simultaneity and real-time & $\begin{array}{l}\text { Personal com- } \\
\text { puter }\end{array}$ & Zigbee \\
\hline 5 & Blood sugar monitoring & $\begin{array}{l}\text { Ability to provide informa- } \\
\text { tion to users }\end{array}$ & Being smart & Smartphone & RFID \\
\hline 6 & Respiratory monitoring & $\begin{array}{l}\text { Ability to be available every- } \\
\text { where }\end{array}$ & Ensuring security and privacy & $\begin{array}{l}\text { Speaker and } \\
\text { microphone }\end{array}$ & GSM \\
\hline 7 & $\begin{array}{l}\text { Electrocardiogram monitor- } \\
\text { ing }\end{array}$ & $\begin{array}{l}\text { Ability to provide social and } \\
\text { emotional support }\end{array}$ & $\begin{array}{l}\text { Use of standards and guide- } \\
\text { lines }\end{array}$ & Landline phone & GPRS \\
\hline 8 & $\begin{array}{l}\text { Body temperature monitor- } \\
\text { ing }\end{array}$ & $\begin{array}{l}\text { Ability to customize the } \\
\text { system }\end{array}$ & $\begin{array}{c}\text { Open and flexible architec- } \\
\text { ture }\end{array}$ & Audio radars & Bluetooth \\
\hline 9 & Diet monitoring & Ability to have a user profile & Use videoconferencing & $\begin{array}{l}\text { Bandwidth } \\
\text { router }\end{array}$ & $\begin{array}{l}\text { Remote phone } \\
\text { line }\end{array}$ \\
\hline 10 & Drug monitoring & Easy to use for the user & $\begin{array}{c}\text { Diagnosis and analysis of } \\
\text { information }\end{array}$ & Webcam & GPS \\
\hline 11 & $\begin{array}{l}\text { Self-assessment of symp- } \\
\text { toms }\end{array}$ & $\begin{array}{l}\text { Ability to provide informa- } \\
\text { tion graphically }\end{array}$ & Use of virtual space (network) & Active hub & LR-WPANs \\
\hline 12 & $\begin{array}{l}\text { Self-reported monitoring } \\
\text { (drowsiness, dizziness, etc.) }\end{array}$ & Ability to motivate & $\begin{array}{l}\text { Having the feature of sending } \\
\text { announcements and news }\end{array}$ & ebook reader & WLAN \\
\hline 13 & - & $\begin{array}{c}\text { Ability to create recommen- } \\
\text { dations }\end{array}$ & $\begin{array}{l}\text { Having the feature of reduc- } \\
\text { ing stored data }\end{array}$ & $\begin{array}{l}\text { Xbee PRO S2B } \\
\text { Module }\end{array}$ & Ethernet \\
\hline 14 & - & $\begin{array}{l}\text { Ability to use different colors } \\
\text { for notifications }\end{array}$ & $\begin{array}{l}\text { Having a set of UPnP pro- } \\
\text { tocols }\end{array}$ & - & MMS \\
\hline 15 & - & Drug management capability & - & - & Cellular network \\
\hline
\end{tabular}

\section{Ethical Considerations}

Compliance with ethical guidelines

This study was approved by the Ethics Committee of the Tehran University of Medical Sciences (Code: 36240-31-02-97).

Funding

The paper was extracted from the $\mathrm{PhD}$. dissertation of the first author, Department of Health Information Technology, Esfarayen Faculty of Medical Sciences, Esfarayen.

\section{Authors' contributions}

All authors equally contributed to preparing this article.

\section{Conflict of interest}

The authors declared no conflict of interest.
Acknowledgments

The authors would like to thank the Department of Health Information Management and the Elderly Research Center of Tehran University of Medical Sciences and all participants. 


\title{
قابليتها و الزامات سامانه يايش از راه دور سلامت سالمندان
}

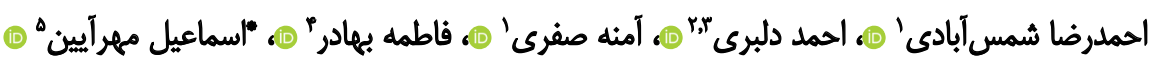

1. كروه فناورى اطلاعات سلامت، دانشكاه علوم يز شكى اسفراين، اسفراين، ايران.

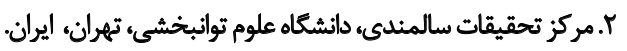

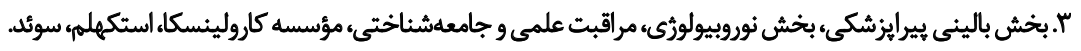

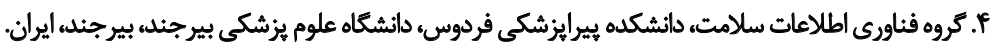

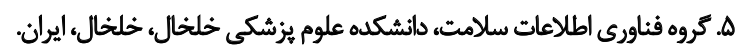

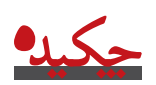

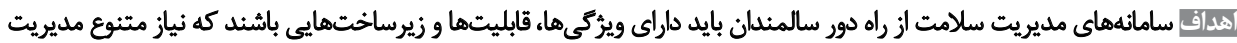

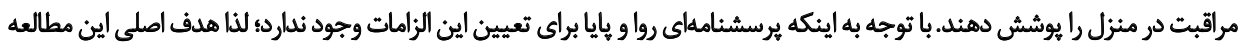

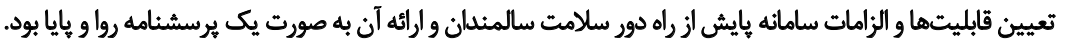

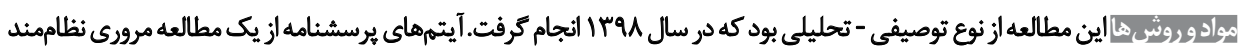

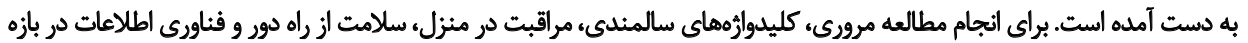

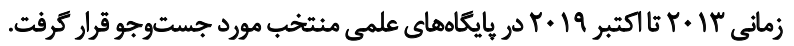

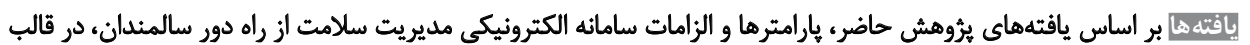

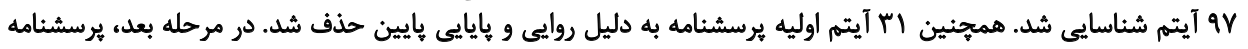

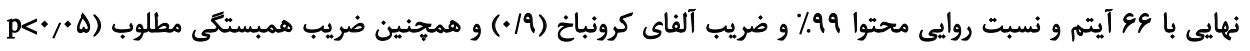
(r=*/AD,

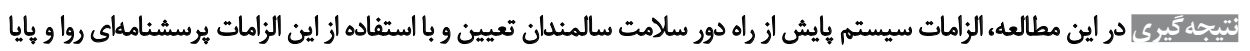

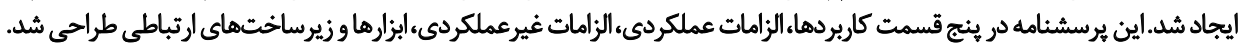

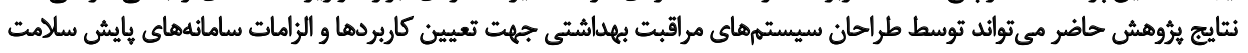

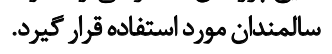

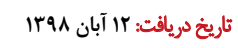

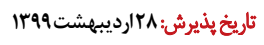

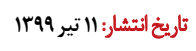

: looglguls

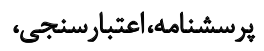
بايش إز راه دور، سلامتيت

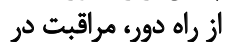
منزل، سالميندان

مداوم و نظارت بر وضعيت سلامت نياز دارند [IT].

doves

در سالهاى اخير دستاوردهاى فناورى اطلاعات و ارتباطات

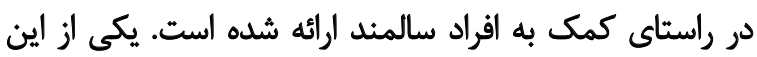

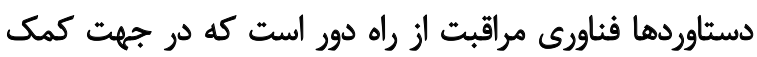

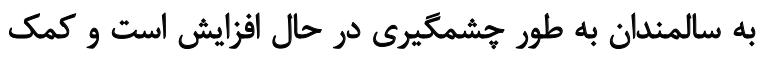

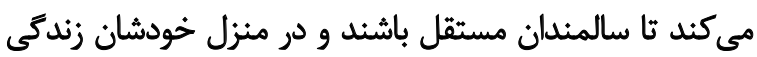

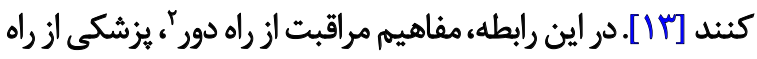

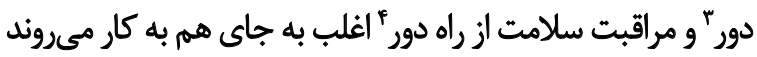

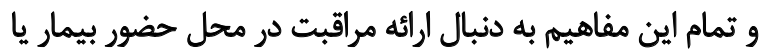

2. Telecare

3. Telemedicine

4. Telehealthcare

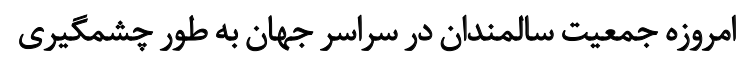

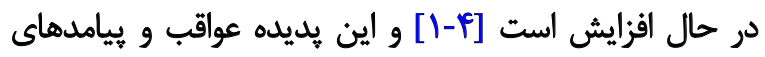

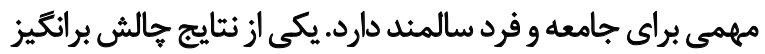

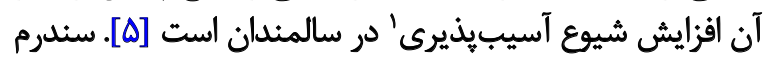

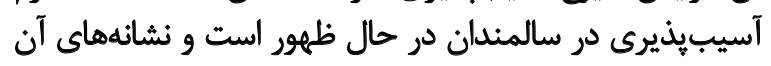

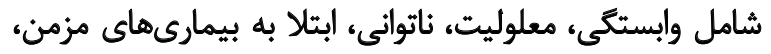

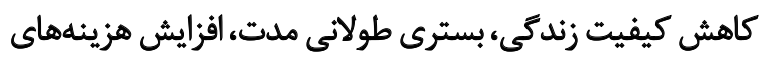

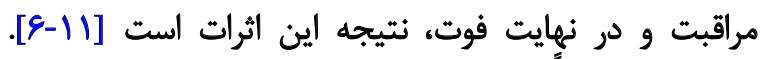

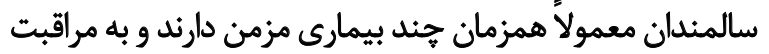

1. Frailty

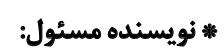

دكتر اسماعيل مهر آيين

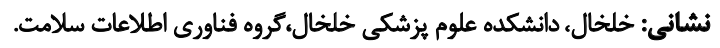
تلفن: بست الكترونيكي: es.mehraeen@gmail.com 
جدول 1. حداقل مقدار CVR قابل قبول بر اساس تعداد خبركان (11)

\begin{tabular}{|c|c|}
\hline حداقل مقدار روايى (\%) & تعداد افراد هانل متخصصصان \\
\hline 99 & $\Delta$ \\
\hline 99 & 8 \\
\hline 99 & $v$ \\
\hline AD & $A$ \\
\hline $\mathrm{vA}$ & 9 \\
\hline RT & 1. \\
\hline$p q$ & 10 \\
\hline Pr & r. \\
\hline$r$ & TA \\
\hline M & r. \\
\hline pq & r. \\
\hline
\end{tabular}

L

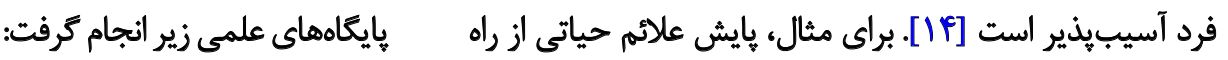

PubMed, Scopus, Web of Science, Google Scholar

بر اساس كليدوارههاى سالمندى، مراقبت در منزل، سلامت

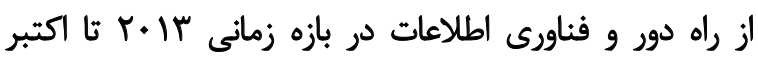

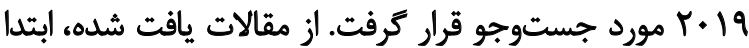

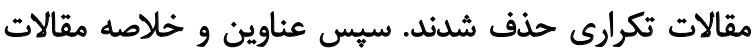

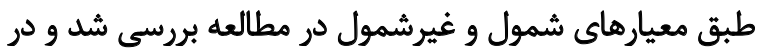

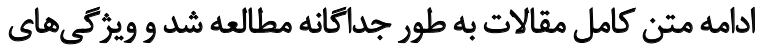
سيستمهاى يايش از راه دور مديريت سلامت سالمندان در منزل

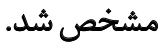

آيتمهاى اطلاعاتى منتج شده از جستوجوى جامع بايكاههاي

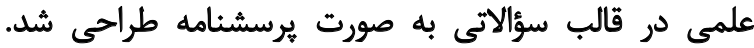

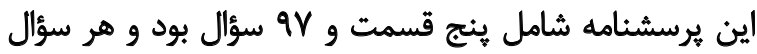
يرسشنامه داراى جهار جواب با عناوين زير تنظيم شد:

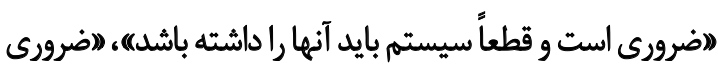

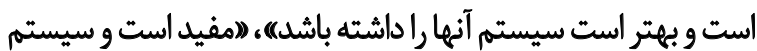

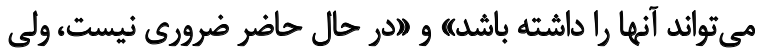

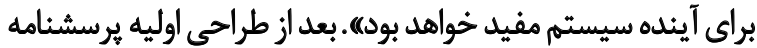

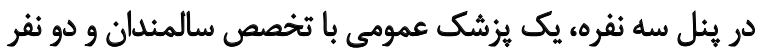
متخصص مديريت اطلاعات، آيتمهاى برسئ برسنامه بررسى و تأيبد شد. بعد از طراحى اوليه برسشنامه به منظور بررسى روايى صورىه

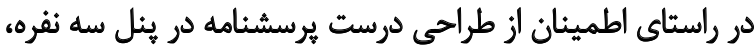

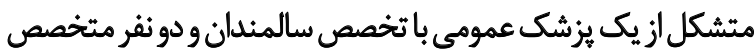

5. Face validity

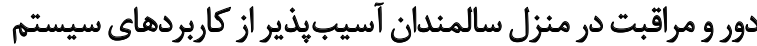

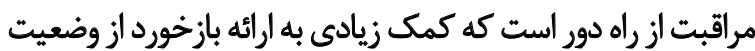

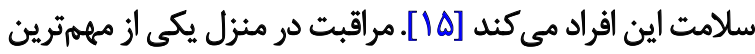

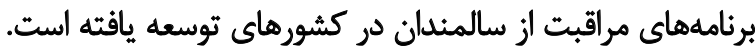

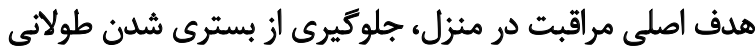

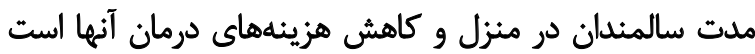

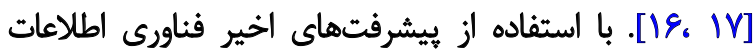

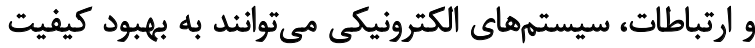

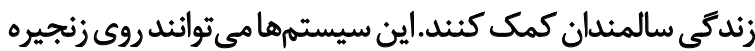

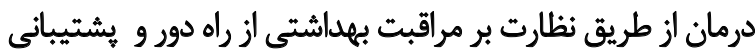
از ارائه خدمات يزشكى در منزل، مؤثر باشئد [1N] با توجه به اهميت بايش از راه دور سلامت سالمندان آسيبيذير در منزل و فقدان ابزارى مناسب در ايران براي تبائ تعيين بارارامترها

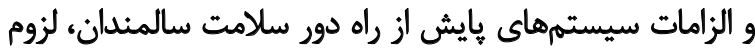

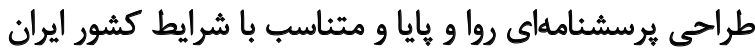

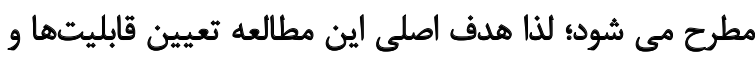

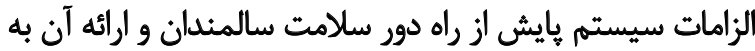
صورت يك يرسشنامه است. روش مطالعه

اين مطالعه از نوع توصيفى - تحليلى بود كه در سال هوبات

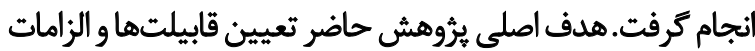

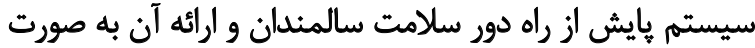

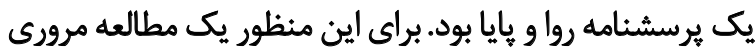

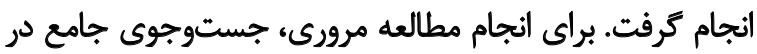




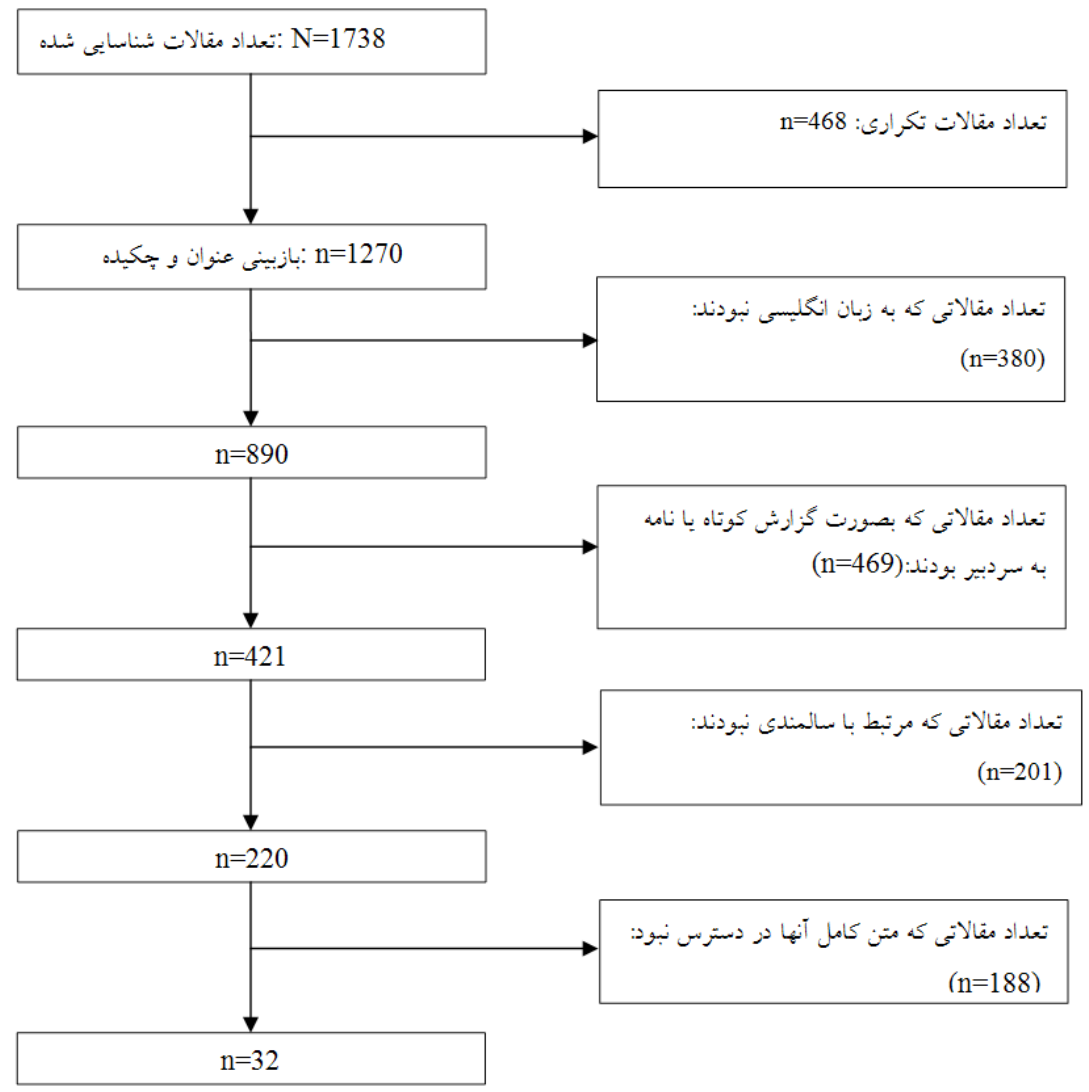

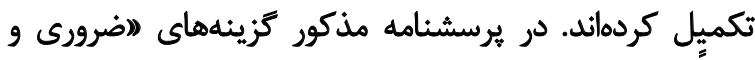

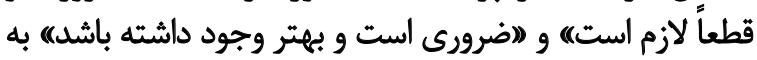

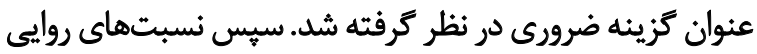

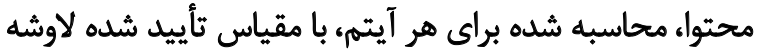

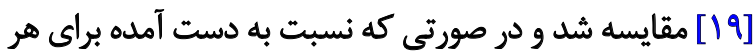

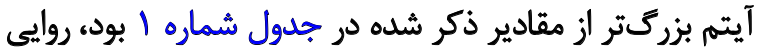
محتواى آن آيتم تأييد شد.

براى آيتمهايي كه نسبت روايي آنها كمتر بودند و از آنجا

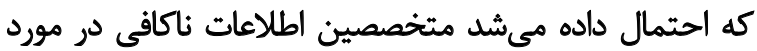

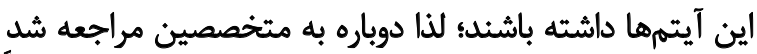

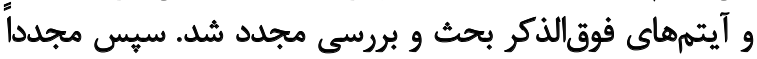

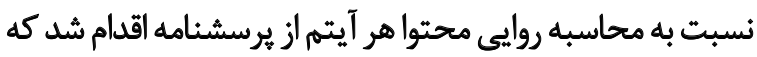
جزئيات آن در جدول شماره ب آمده است.

\section{نحوه محاسبه بايائيى}

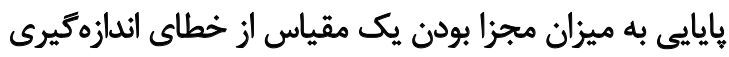

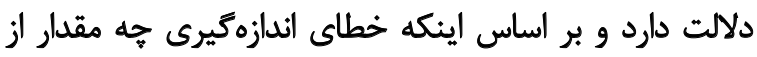

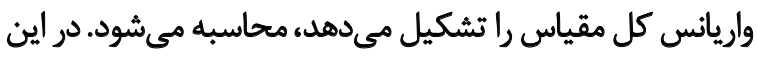

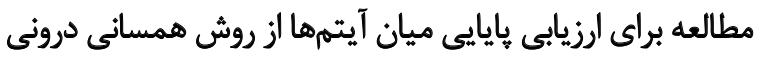

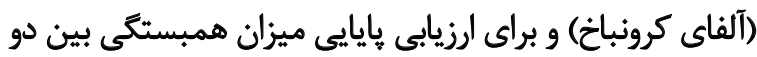

مديريت اطلاعات سلامت، آيتمهاى يرسشنامه بررسى و تأييد شد.

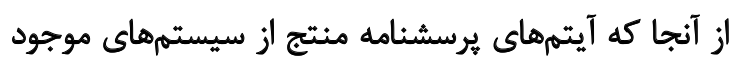

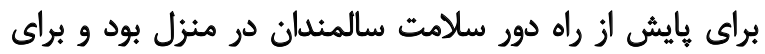

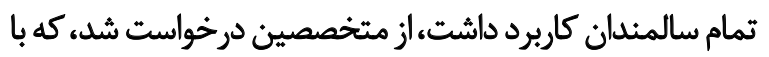

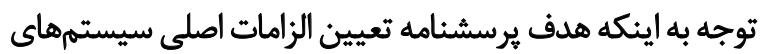

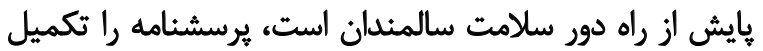

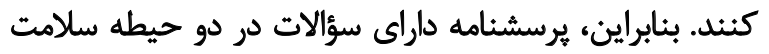

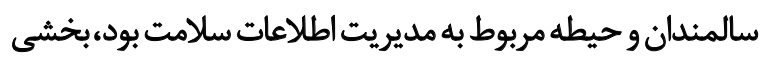

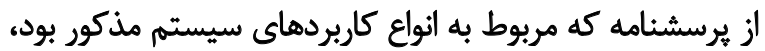

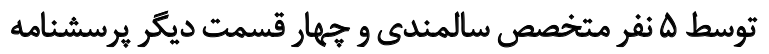

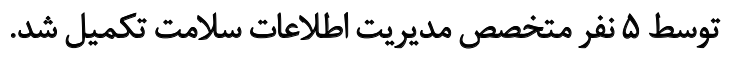
براى بررسى روايى، يرسشنامه توسط متخصصين تكميل شده بـ بردي

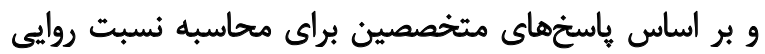

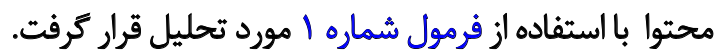

$C V R=\frac{n e_{-} n / 2}{n / 2}$

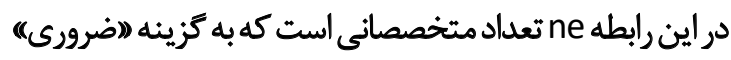

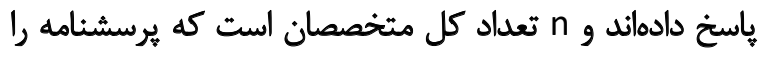


جدول r. كل آيتمهاى بيثنويس يرسشئامه

\begin{tabular}{|c|c|c|c|c|}
\hline زيرساخت ارتهائى & انواع ابزارها & غيرعملكردى & الزامات عملكردى & انواع كاربردها \\
\hline اينترنت & سنسورهاى بالينى & دانتن بإيكاه داده مركزى اطلاعات & ارائه آموزش به كاربران & يايش فُشار خُون \\
\hline واىفاى و وايمكس & دوربين & تشخيص و تحليل اطلاعات & تشخيص ميزان اورزانسى بودن & بايش ضربان قلب \\
\hline 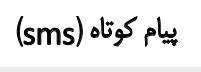 & تبلت & كاربر بيسندى & اراثه يادآورى & بايش سطح اكسيرن اشباع شده \\
\hline Zigbee & رايائه شخصصى & يرونده بالينى شخصى & قابليت ارائه هشدار & يايش ونن \\
\hline RFID & كوشى هوشمثد & هبتنى برو وب بودن & قابليت اراثه نمايش اطلاعات به & هايش قندل خُون \\
\hline GSM & و ل مبكم & دارا بودن محيط مجازى & قابليت همه جادر دسترس بودن & بايش تنفس \\
\hline GPRS & اسييكر و ميكروفن & همزمائى و بلادريك بودن & قابليت حمايت اجثماعى و احساسى & بايش الكتروكارديوكرام \\
\hline Bluetooth & إزار Intel health guide & هوشُمندى & قابليت شخصى سازى سيسثم & بايش تحركات بلنى \\
\hline $\begin{array}{l}\text { Telephone } \\
\text { line }\end{array}$ & كؤشى تلفن ثابت & تأمين امنيت و حريم خصوصى & قابليت داشتن يروفايل كاربر & هايش دماي بلن \\
\hline GPS & 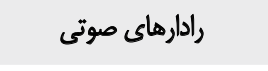 & داشتن مديريت إيليكيشنها & قابليت استفاده آسان براى كاربر & تشخيص انعقاد خون \\
\hline LR-WPANs & روتر بهناى بائد & استفاده از استانداردها و كايدلاينها & قابليت ارائه اطاطلاعات به صورت & تشخيص بي قرارى \\
\hline WLAN & كتابخوان الكترونيكى & استفاده از أنواع ايليكيشنها & ق قابليت ايجاد انكيزه & بايش مصرف دارو \\
\hline Ethernet & كنترل كننده (دسته) بازي & استفاده از ويلئوكنفراثس & قابليت اجراى بازى هاى رايانهاى أز & بايش اميدانس قفسه سينه \\
\hline MMS & هاب فعال & استفاده از وب يورتال & قابليت أرائه راهنمايى به صورت & تشخيص ياسخ كالوائيزه يوست \\
\hline \multirow[t]{13}{*}{$\begin{array}{l}\text { Cellular } \\
\text { network }\end{array}$} & ستسور تشخيص دهنده & استفاده از لينك ويدئويى & 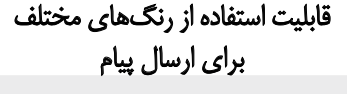 & بايش الكتروميوكرافي \\
\hline & هاب فعال & استفاده از ييام صوتى & قابليت نمايش دورماى اطلاعات & هايش رزيم غذايي \\
\hline & RFID دستكاه خواثنين & قابليت استثاده از نمودارهاي أيميمشنى & قابليت ايجاد توصيه براى كاربران & 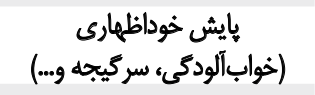 \\
\hline & 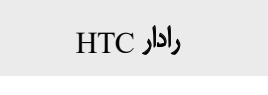 & دائن ويزكى كاهش دادهاى زخيره & قابليت مديريت دارو & بايش وضعيت روحى و روانى \\
\hline & & داشتن ويزّكى ارسال نوتيفيكيشن & قابليت استفاده از Google TV & \\
\hline & & اسثفاده از حذمات فضضاى ابرى & & \\
\hline & & SNOMED CT استفاده از & & \\
\hline & & ارتباط مستقيه با HIS بيمارستان & & \\
\hline & & دانشن معمارى باز و انعطاف يذير & & \\
\hline & & بر بايه عامل بودن & & \\
\hline & & UPnP داشت مجموعه يروتكل هاى & & \\
\hline & & POP - UP دئن ويزّى & & \\
\hline & & استفاده از يروتكل HTTPS براى & & \\
\hline
\end{tabular}


انجام شده است. اين مطالعه با هدف تعيين قابليتها و الزامات

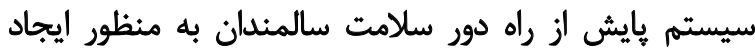

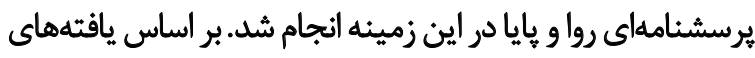

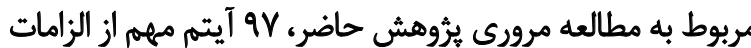

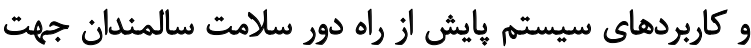

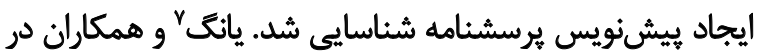

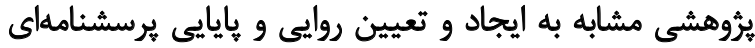

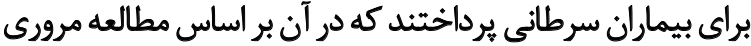

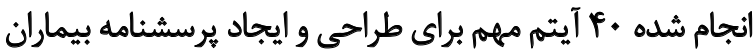

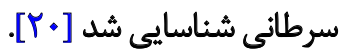

بايد توجه داشت كه شناسايى الزامات يك سيسته به تنهايى

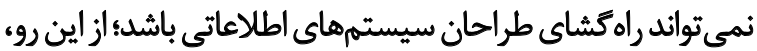

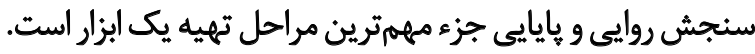

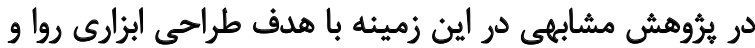

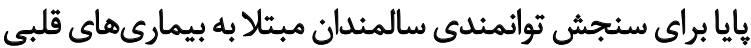

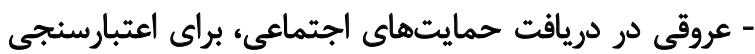

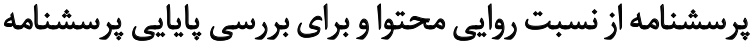
از آزمون - بازآزمون و آلفاى كرونباخ استفاده شدي

نتايج اين مطالعه مشابه نشان داد كه از ها آيتم اوليه

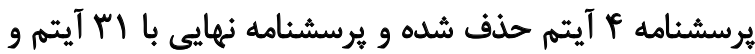

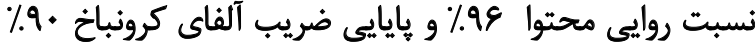

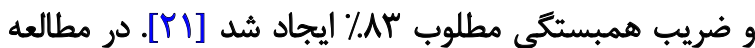

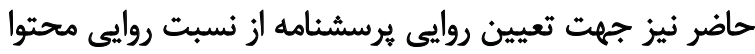

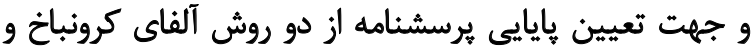

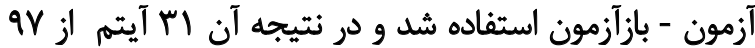

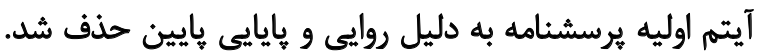

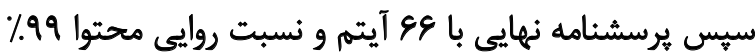

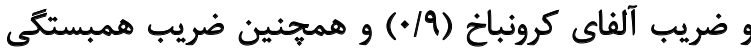
مطلوب (r=0.85, P<0.05) ايجاد شد.

مطابق نتايج يُؤوهش حاضر، در يرسشنامه نهايى آيتمهاي

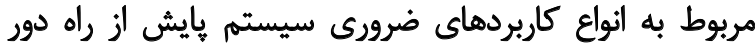

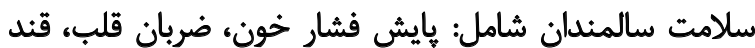

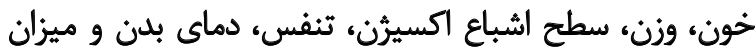

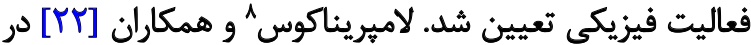

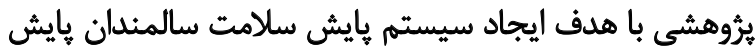

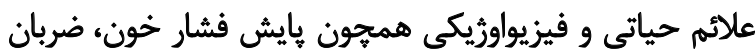

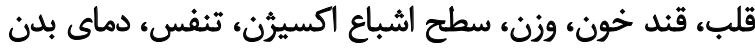

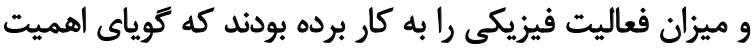

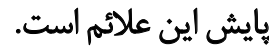

در يثروهش حاضر آيتمهاى ضرورى مربوط به الزامات عملكردى

7. Young

8. Lamprinakos
اجزاي يك مقياس با يك فاصله زمانى و مكانى از روش آزمون بازآزمون (ضريب اسييرمن - براوان) استفاده شداني

يافتها

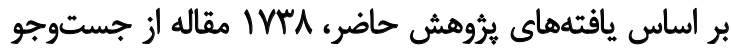

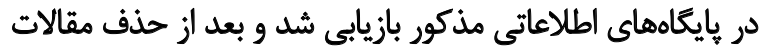

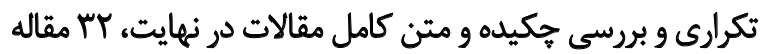

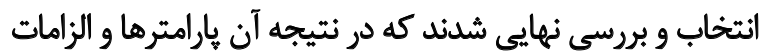

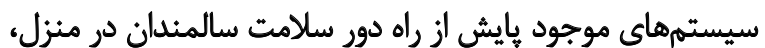

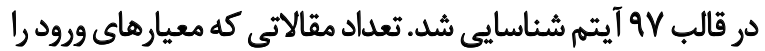
نداشتند در نمودار يريزما 'نشان داده شده است (تصوير شماره ()). بر اساس الزامات شناسايى شده، يرسشنامه اوليه در ينج

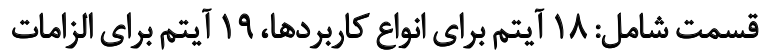

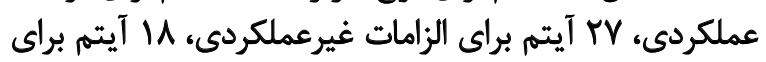

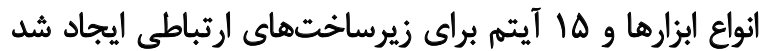

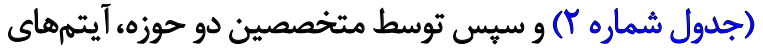
يرسشنامه بررسى و تأييد شد.

نتايج محاسبه روايى محتوا در كام اول نشان داد، از لو آيتم

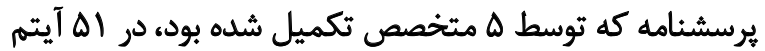

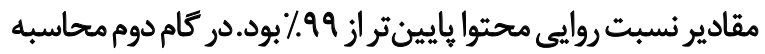

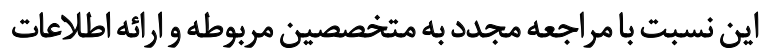

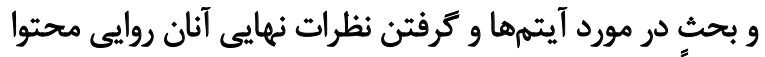

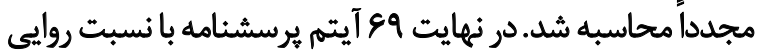

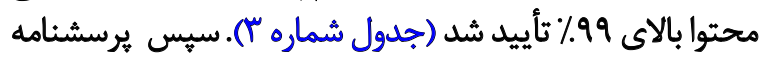

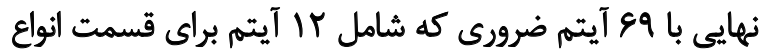

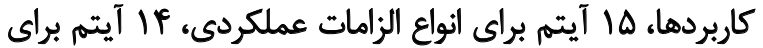

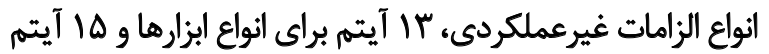

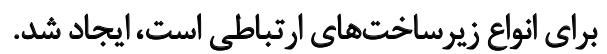

به منظور بررسى يايايى درونى يرسشنامه از ضريب آلفاى كرونباخ

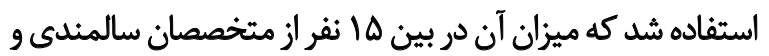

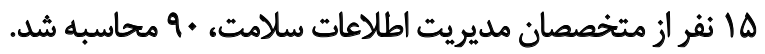

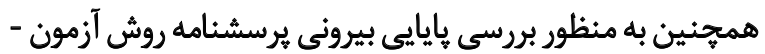

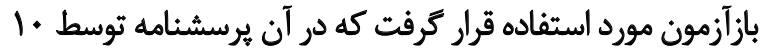

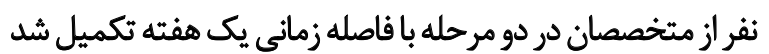

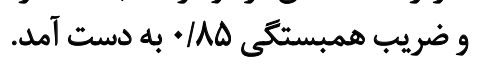

بحث

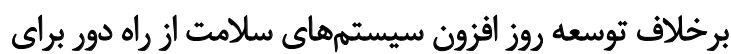

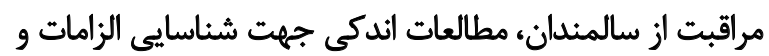

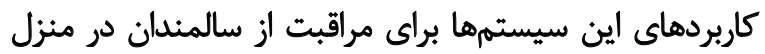

6. PRISMA flow diagram depicting the exclusion and inclusion of articles 


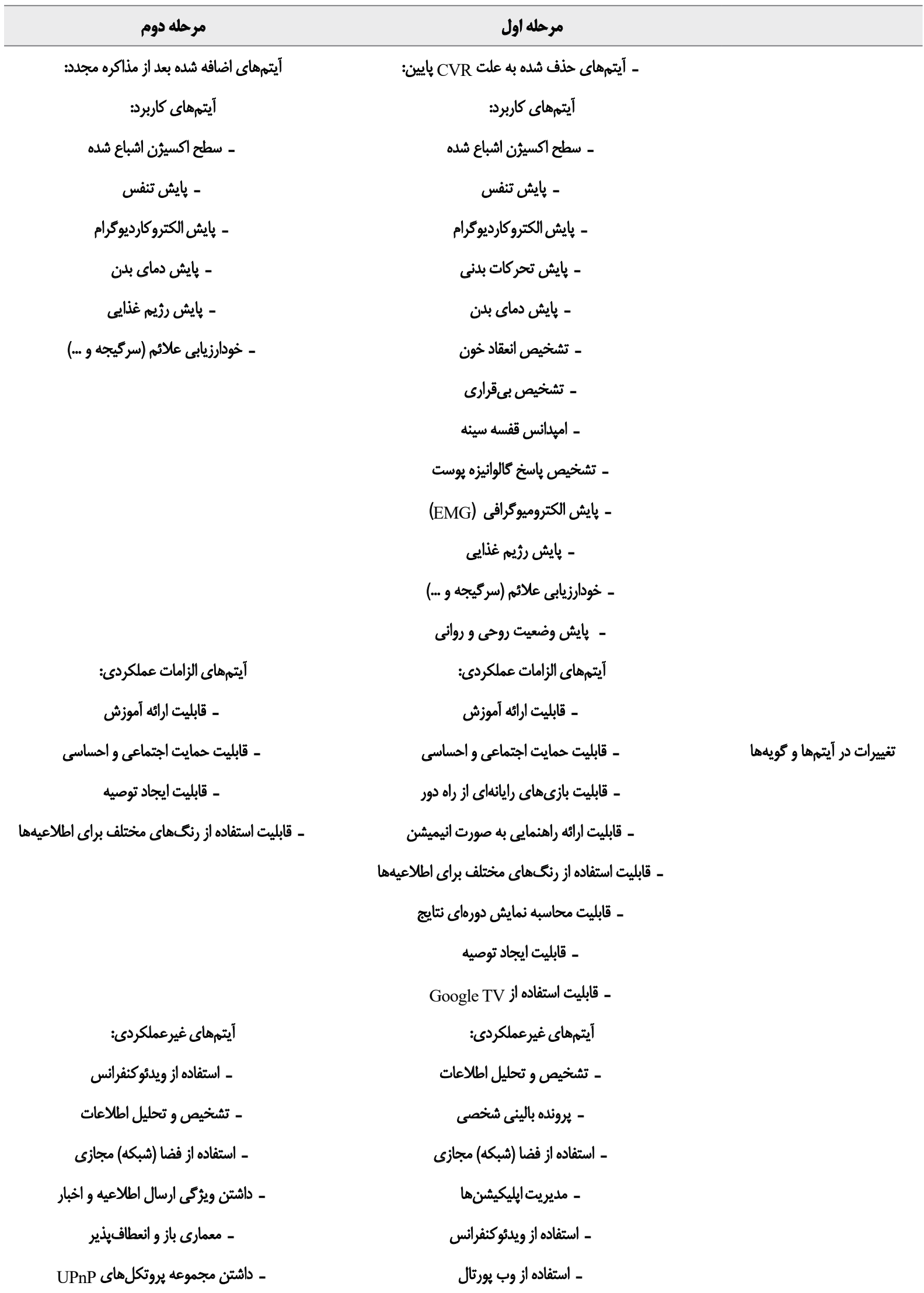




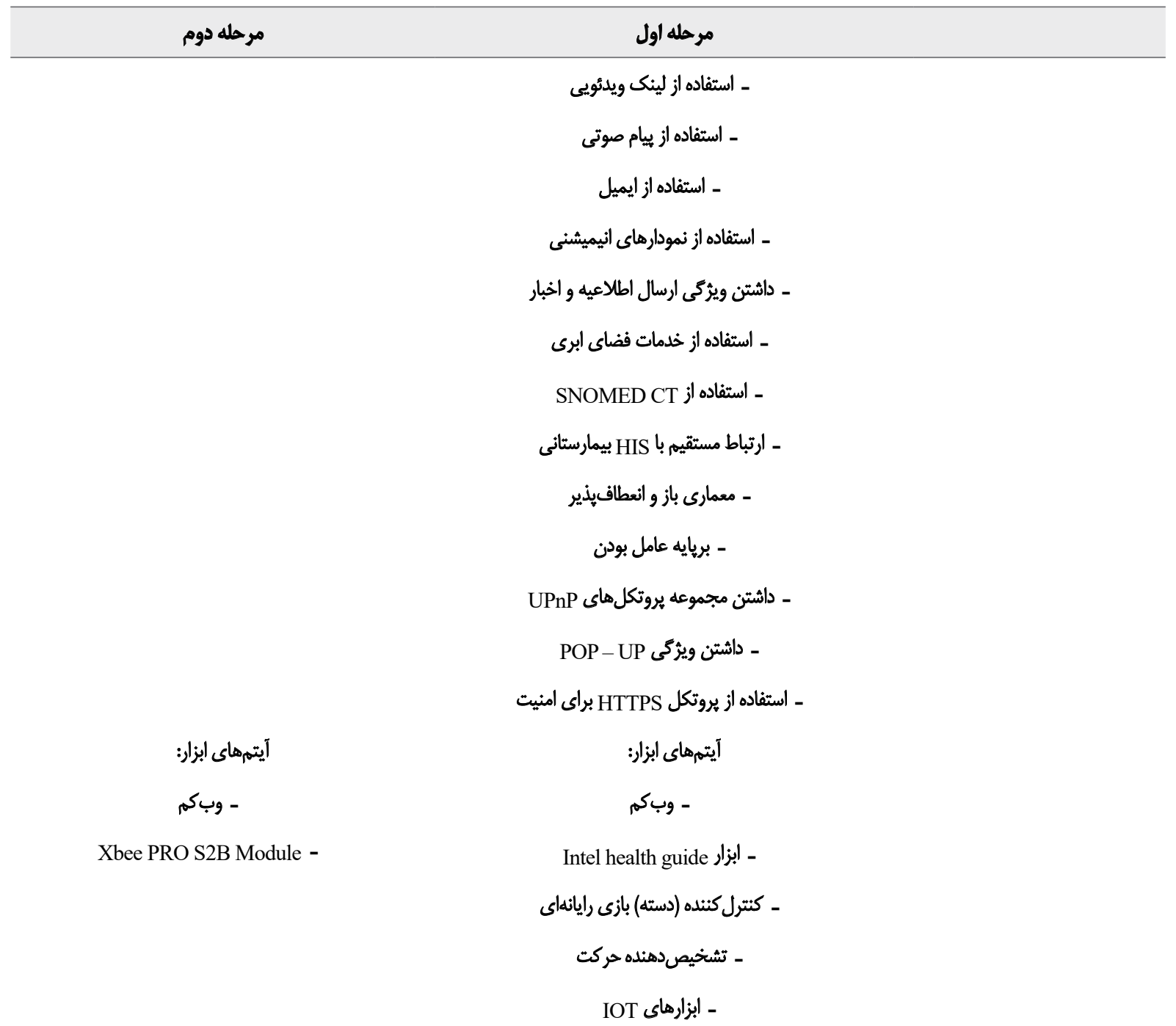

Xbee PRO S2B Module -

HTC راs -

آيتمهاي زيرساخت ارتباطى:

آيتمهاي زيرساخت ارتباطي:

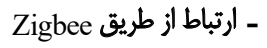

- ارتباط از طريق Zigbee

- ارتياط از طريق RFID

- ارتباط از طريق RFID

- ارتباط از طريق LR-WPANs

LR-WPANs ارتباط از طريق

- ارتباط از طريق Cellular network

- ارتباط از طريق Cellular network

$1 \% / M=1$

أيتهمهاى كاربرد:

$\mid q / \backslash 8=1$

أيتمهاى الزامات عملكردى: ז'.

$|q /| q=1$

آيتمهاى الزامات غيرعملكردى:

ميانكين CVR سازها

$11 / 11=1$

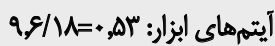

$1 \Delta / 1 Q=1$

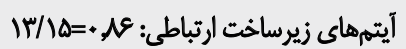




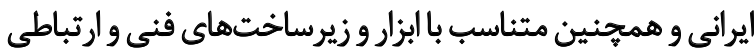

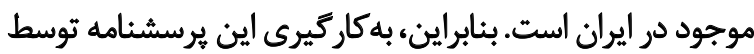

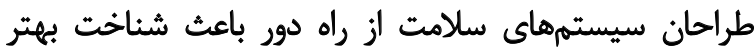

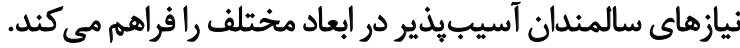

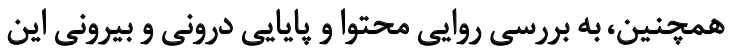

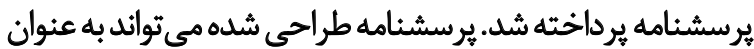

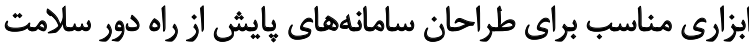

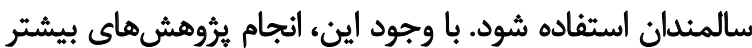

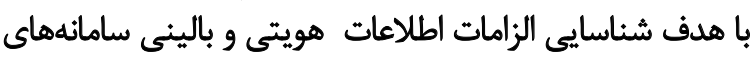
جايش از راه دور سلامت سالمندان ضرورى است التات

از محدوديتهاى اين مطالعه، مي توان به حجم نمونه غايين

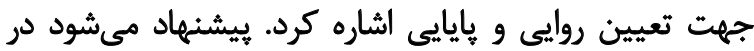

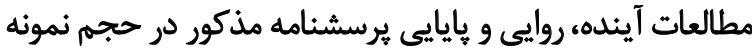

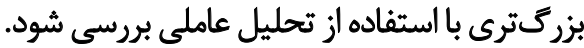

ماحظات اخلاقي

\section{بيروى أو اصول الخاق ثرؤش}

تمامى اصول اخلاقى اين تحقيق توسط دانشكاه علوم يزشكى

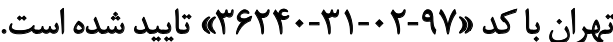

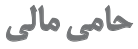

اين مقاله حاصل پايان نامه دكتراي نويسنده اول در گروه فناورى اطلاعات سلامت، دانشكده علوم يزشكي اسئ اسفراين، اسفراين است. - است اطلات

$$
\text { مشاركت نويسند كَان }
$$

تمام نويسندكان در آمادهازى اين مقاله مشاركت داشتهاند.

$$
\text { تعارض مناقع }
$$

بنابر اظهار نويسندكان، اين مقاله تعارض منافع ندارد.

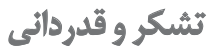

بدينوسيله نويسندكان مراتب تشكرو قدردانى رالز گروه مديريت

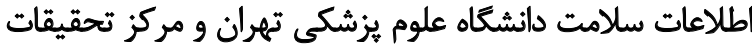

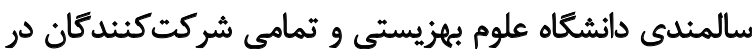

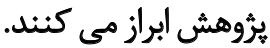

براي سيستم هايش از راه دور سلامت سالمندان آسيبيذير در منزل

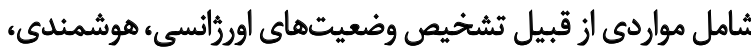

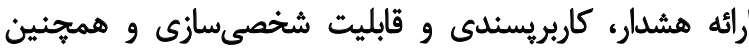

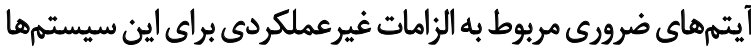

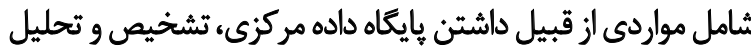

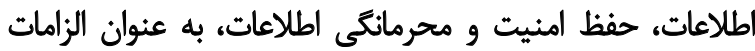

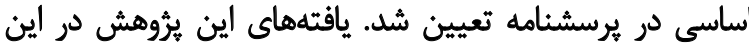

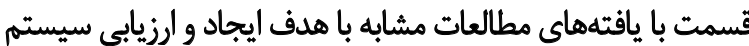

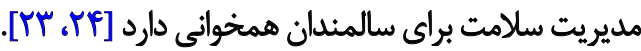

همجنين، در مطالعه حاضر، ابزارهاي ضرورى جهت به كاركيرى

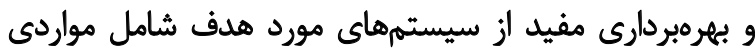

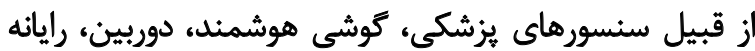

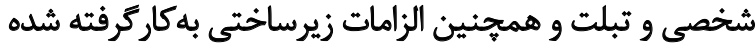
در اين سيستمها شامل مواردى از قبيل اينترنت، بلوتوث، وائين

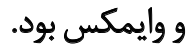

در مطالعهاى كه توسط حسين و همكاران با هدف طراحي

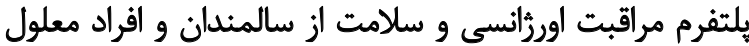

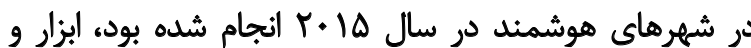

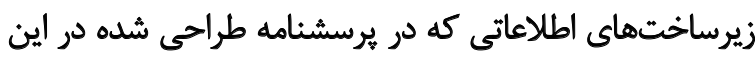

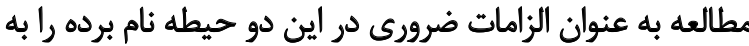

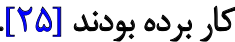

بنا به نظر جامعه آمارى يُروهش حاضر، از آنجا كه كروه هدف

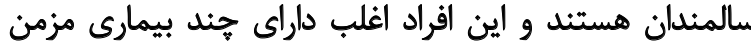

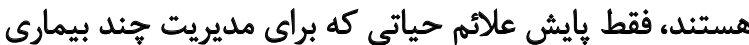

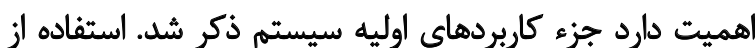

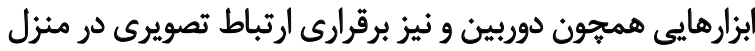

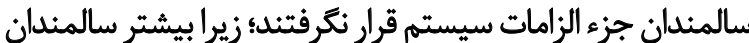

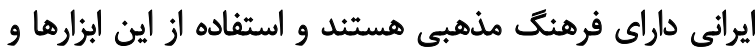

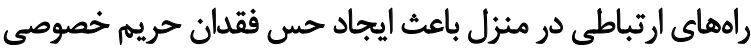

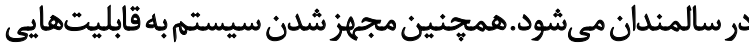

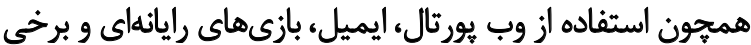

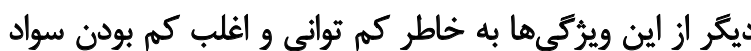

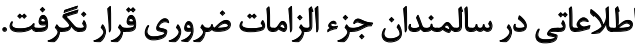

\section{تتيجه كيرى نهايى}

در اين مطالعه قابليتها و الزامات سيستم :ايش از راه داه دور

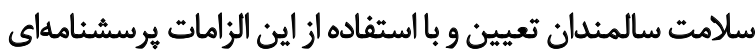

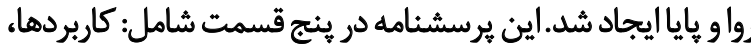

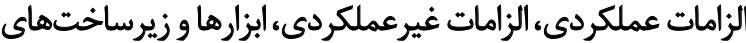

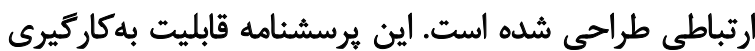

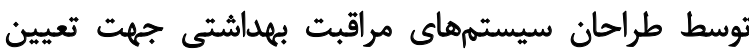

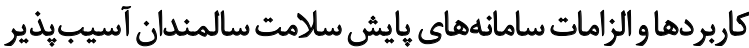

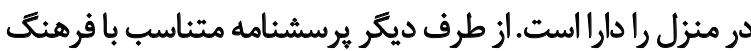




\section{References}

[1] Arif MJ, El Emary IM, Koutsouris DD. A review on the technologies and services used in the self-management of health and independent living of elderly. Technology and Health Care. 2014; 22(5):677-87. [DOI:10.3233/THC-140851] [PMID]

[2] Gaddam A, Mukhopadhyay SC, Gupta GS. Elder care based on cognitive sensor network. IEEE Sensors Journal . 2011; 11(3):57481. [DOI:10.1109/JSEN.2010.2051425]

[3] Liu L, Stroulia E, Nikolaidis I, Miguel-Cruz A, Rios Rincon A. Smart homes and home health monitoring technologies for older adults: A systematic review. International Journal of Medical Informatics. 2016; 91:44-59. [DOI:10.1016/j.ijmedinf.2016.04.007] [PMID]

[4] Hamdi O, Chalouf MA, Ouattara D, Krief F. eHealth: Survey on research projects, comparative study of telemonitoring architectures and main issues. Journal of Network and Computer Applications. 2014; 46:100-12. [DOI:10.1016/j.jnca.2014.07.026]

[5] Andreasen J, Lund H, Aadahl M, Gobbens RJJ, Sorensen EE. Content validation of the Tilburg Frailty Indicator from the perspective of frail elderly. A qualitative explorative study. Archives of Gerontology and Geriatrics. 2015; 61(3):392-9. [DOI:10.1016/j. archger.2015.08.017] [PMID]

[6] Romero-Ortuno R, Walsh CD, Lawlor BA, Kenny RA. A frailty instrument for primary care: Findings from the Survey of Health, Ageing and Retirement in Europe (SHARE). BMC Geriatrics. 2010; 10:57. [DOI:10.1186/1471-2318-10-57] [PMID] [PMCID]

[7] Clegg A, Young J. The frailty syndrome. Clinical Medicine. 2011; 11(1):72-5. [DOI:10.7861/clinmedicine.11-1-72] [PMID] [PMCID]

[8] Wou F, Conroy S. The frailty syndrome. Medicine. 2013; 41(1):135. [DOI:10.1016/j.mpmed.2012.10.004]

[9] Xue QL. The frailty syndrome: Definition and natural history. Clinics in Geriatric Medicine. 2011; 27(1):1-15. [DOI:10.1016/j. cger.2010.08.009] [PMID] [PMCID]

[10] Clegg A, Young J, Iliffe S, Rikkert MO, Rockwood K. Frailty in elderly people. The Lancet. 2013; 381(9868):752-62. [DOI:10.1016/ S0140-6736[12)62167-9]

[11] Peters LL, Boter H, Buskens E, Slaets JP. Measurement properties of the Groningen Frailty Indicator in home-dwelling and institutionalized elderly people. Journal of the American Medical Directors Association. 2012; 13(6):546-51. [DOI:10.1016/j. jamda.2012.04.007] [PMID]

[12] Urban M. 'This really takes it out of you!' The senses and emotions in digital health practices of the elderly. Digital Health. 2017; 3. [DOI:10.1177/2055207617701778] [PMID] [PMCID]

[13] Stowe S, Harding S. Telecare, telehealth and telemedicine. European Geriatric Medicine. 2010; 1(3):193-7. [DOI:10.1016/j. eurger.2010.04.002]

[14] Barlow J, Singh D, Bayer S, Curry R. A systematic review of the benefits of home telecare for frail elderly people and those with long-term conditions. Journal of Telemedicine and Telecare. 2007; 13(4):172-9. [DOI:10.1258/135763307780908058] [PMID]

[15] Coyte PC, McKeever P. Home care in Canada: Passing the buck. Canadian Journal of Nursing Research Archive. 2016; 33(2):11-25. https://cjnr.archive.mcgill.ca/article/view/1631
[16] Cronfalk BS, Fjell A, Carstens N, Rosseland LMK, Rongve A, Rönnevik DH, et al. Health team for the elderly: A feasibility study for preventive home visits. Primary Health Care Research \& Development. 2017; 18(3):242-52. [DOI:10.1017/ S1463423617000019] [PMID]

[17] Bobillier Chaumon ME, Michel C, Tarpin Bernard F, Croisile B. Can ICT improve the quality of life of elderly adults living in residential home care units? From actual impacts to hidden artefacts. Behaviour \& Information Technology. 2014; 33(6):574-90. [DOI:1 0.1080/0144929X.2013.832382]

[18] Lupton D. Editorial: Towards sensory studies of digital health. Digit Health. 2017; 3:2055207617740090. [DOI:10.1177/2055207617740090] [PMID] [PMCID]

[19] Lawshe $\mathrm{CH}$. A quantitative approach to content validity. Personnel Psychology. 1975; 28(4):563-75. [DOI:10.1111/j.1744-6570.1975.tb01393.x]

[20] Young JM, Walsh J, Butow PN, Solomon MJ, Shaw J. Measuring cancer care coordination: Development and validation of a questionnaire for patients. BMC Cancer. 2011; 11:298. [DOI:10.1186/1471-2407-11-298] [PMID] [PMCID]

[21] Musavinasab M, Ravanipour M, Pouladi Sh, Motamed N, Barekat M. [Examining the validity and reliability of the cardiovascular disease questionnaire in measuring the empowerment of elderly patients to receive social support (Persian)]. Salmand: Iranian Journal of Ageing. 2016; 11(2):258-69. [DOI:10.21859/ sija-1102258]

[22] Lamprinakos GC, Asanin S, Broden T, Prestileo A, Fursse J, Papadopoulos KA, et al. An integrated remote monitoring platform towards telehealth and telecare services interoperability. Information Sciences. 2015; 308:23-37. [DOI:10.1016/j.ins.2015.02.032]

[23] Dhillon JS, Wünsche B, Lutteroth C. Designing and evaluating a patient-centred health management system for seniors. Journal of Telemedicine and Telecare. 2016; 22(2):96-104. [DOI:10.1177/1357633X15586082] [PMID]

[24] Saponara S, Donati M, Bacchillone T, Sanchez-Tato I, Carmona C, Fanucci L, et al. Remote monitoring of vital signs in patients with chronic heart failure: Sensor devices and data analysis perspective. Paper: presented at: 2012 IEEE Sensors Applications Symposium Proceedings. 7-9 February 2012; Brescia, Italy. [DOI:10.1109/SAS.2012.6166310]

[25] Hussain A, Wenbi R, da Silva AL, Nadher M, Mudhish M. Health and emergency-care platform for the elderly and disabled people in the Smart City. Journal of Systems and Software. 2015; 110:253-63. [DOI:10.1016/j.jss.2015.08.041] 